Check for updates

Cite this: RSC Adv., 2019, 9, 20185

Received 21st May 2019

Accepted 24th June 2019

DOI: 10.1039/c9ra03837j

rsc.li/rsc-advances

\section{Controllable fluorescence via tuning the $m$ - substituents of added aromatic molecules in a pyrene derivative-decorated porous skeleton $\uparrow$}

\begin{abstract}
Jianbin Wu, Songyang Huang, Xi Wang (D) * and Ming Bai (D)
A novel pyrene derivative based composite fluorescent material was developed by immobilizing the pyrene1-carboxylic acid (PyCOOH) into the pores of porous polyurea microspheres (denoted as $\mathrm{PyCOOH}$ decorated PPUM). Encouragingly, the fluorescence spectrum of this synthesized composite microsphere only exhibited monomer emission of guest $\mathrm{PyCOOH}$, indicating that the porous skeleton PPUM has excellent isolation ability to separate the guest molecules from each other. This discovery will provide an effective strategy to design and synthesize pyrene based host-guest systems without excimer emission. Notably, the PyCOOH-decorated PPUM can keep good fluorescent stability when dispersed in many organic solvents. More excitingly, it was found for the first time that the fluorescence of such a material can be regulated by adding aromatic compounds containing different $m$-substituted groups. When $m$ cresol was added, the intensity of the monomer emission enhanced significantly due to the unusual dissolution of the host porous polyurea sphere. By adding the $m$-toluidine, the monomer emission without the fluorescence of unassociated $\mathrm{PyCOOH}$ increased owing to the connection of $m$-toluidine and $\mathrm{PyCOOH}$ which escaped from the pores. In the presence of $m$-methylacetophenone and $m$-toluic acid, the monomer emission showing different degrees of decline was observed respectively because of the different substitution process. This result will contribute to the exploration of more promising candidates for pyrene-based fluorescent sensors.
\end{abstract}

\section{Introduction}

Pyrene derivatives have drawn tremendous attention due to their intriguing dual emissive nature: one for the monomer emission (370-430 $\mathrm{nm}$ ) and another for the excimer emission around $480 \mathrm{~nm}$. Various pyrene based materials exhibit diverse promising applications in biolabelling, chemosensing, protein detection and so on. ${ }^{\mathbf{1 - 1 4}}$ Recently, great endeavours have been devoted to encapsulating the pyrene moiety in a closed space to block the formation of excimers, ${ }^{15}$ because the excimer emission sometimes disturbs quantitative analysis when using a pyrene based probe. Pyrene-bridged macrocage molecules were synthesized to inhibit the formation of the excimer, in which the pyrene moiety is sterically protected by outside alkyl chains. ${ }^{16}$ This direct strategy will exhibit broad prospects, but still faces challenges, because it is quite difficult to avoid the

Marine College, Shandong University, Weihai, Weihai 264209, People's Republic of China.E-mail: xi_wang@sdu.edu.cn

$\dagger$ Electronic supplementary information (ESI) available: The details of the reagents used in the synthesis; synthetic route of PPUM; UV-Vis absorption spectra; powder XRD patterns; XPS spectra; SEM images in the presence of the solution with different $\mathrm{pH}$; IR spectra in the absence and presence of $\mathrm{m}$-cresol; SEM and fluorescence spectra of PyCOOH-decorated PPUM upon addition of various solvents and aromatic compounds respectively. See DOI: 10.1039/c9ra03837j generation of large amounts of the non-caged isomer as byproduct. Therefore, another facile and effective method, such as adsorbing the guest molecule in surfactant-intercalated graphite oxide (GO), has been developed to obtain the monomer emission. ${ }^{17}$ Comparing with 2-D GO layers, there appears a strong possibility that the 3-D uniform porous skeleton will exhibit better isolation ability to separate the guest molecules from each other, however, the encapsulation of the pyrene derivative into such host is rarely reported. Therefore, it motivate us to carry out the study on designing the novel pyrenedecorated composite material showing no excimer emission by using a porous structure as host for the isolation of pyrene derivative.

As a promising 3-D porous host skeleton, the porous polyurea microsphere (PPUM) is of great importance because it can be widely used in the host-guest system. Although several approaches were devoted to achieve the high production efficiency for the preparation of polyurea microspheres, ${ }^{18-24}$ the syntheses and applications of the PPUM with uniform pores have been less investigated. Recently, a new crosslinked PPUM with the potential application for water treatment were successfully prepared by adding the triethylenetetramine (TETA) as a crosslinking agent and monodisperse $\mathrm{SiO}_{2}$ particles as porogen via facile one-step precipitation polymerization. ${ }^{25}$ Since the PPUM showed excellent chemical stability, exhibited 
controllable pores, as well as contained urea group and amine group which probably facilitated to attach the acid molecules by hydrogen bonding or electrostatic interactions, it was chosen as a host to load the fluorescent guest pyrene-1-carboxylic acid (PyCOOH). ${ }^{26}$ Interestingly, this prepared $\mathrm{PyCOOH}$-decorated PPUM exhibited no excimer emission of pyrene derivative. This material can also keep good fluorescent stability when dispersed in many common organic solvents, especially in ethyl acetate. Encouragingly, it was found that the significantly fluorescence changes were induced by adding various $m$ substituted aromatic compounds ( $m$-cresol, $m$-toluidine, $m$ toluic acid and $m$-methylbenzoate). Comparing with the normally established liquid chromatography and mass spectrometry, these aromatic compounds were rarely determined utilized the fluorescent pyrene-based material. ${ }^{27}$ The possible mechanisms of the different fluorescent responses by adding these aromatic compounds were further investigated.

\section{Experimental}

\section{Materials and methods}

All the details of the source materials used in the synthesis which were purchased from Energy Chemical were listed in Table S1 of ESI. $\dagger$ Powder X-ray diffraction (XRD) data were collected on a Rigaku D/Max 2550 X-ray diffractometer with $\mathrm{Cu}$ $\mathrm{K} \alpha$ radiation $(\lambda=0.15418 \mathrm{~nm})$. Fourier transform-infrared (FTIR) spectra of the solids were recorded on a Nicolet Impact 410 FT-IR spectrometer using the $\mathrm{KBr}$ pellet technique. X-ray photoelectron spectroscopy (XPS) measurements were performed using a Thermo Escalab 250 spectrometer with monochromatized $\mathrm{Al} \mathrm{K} \alpha$ excitation. The morphology of the related particles was investigated on a JEOL-2100 transmission electron microscope (TEM) and a Nova NanoSEM 450 scanning electron microscope (SEM). The studies of the UV-Vis spectra were performed on a PerkinElmer UV-Vis instrument. The emission spectra of the samples were detected on a Hitachi F-7000 spectrofluorometer.

\section{Synthetic procedures}

In the typical synthesis of $\mathrm{SiO}_{2}$ nanoparticles, $5 \mathrm{~mL}$ of the ammonium hydroxide was mixed in $50 \mathrm{~mL}$ ethanol, and $1.5 \mathrm{~mL}$ of tetraethoxysilane was dropwise added into the mixture. ${ }^{28}$ The above solution was stirred in a three necked flask at $40{ }^{\circ} \mathrm{C}$ using the oil bath for $12 \mathrm{~h}$. The resulted nanoparticles were separated by centrifugation, washed with ethanol and deionized water, and dried at $70{ }^{\circ} \mathrm{C}$ in an oven. The $\mathrm{PyCOOH}$-decorated porous polyurea microspheres were prepared according to a modified synthesis method of the non-crosslinked PPUMs, $2.5 \mathrm{~g}$ of the isophorone diisocyanate (IPDI) and $0.5 \mathrm{~g}$ of above prepared $\mathrm{SiO}_{2}$ nanoparticles were mixed in $50 \mathrm{~g}$ of $\mathrm{H}_{2} \mathrm{O}$-acetone mixture with $3 / 7$ mass ratio. ${ }^{25}$ The homogeneous mixture kept stirring at $30{ }^{\circ} \mathrm{C}$ for $4 \mathrm{~h}$ using the oil-bath system. The resulted polyurea/ $\mathrm{SiO}_{2}$ composite microspheres were separated by centrifugation and washed with $\mathrm{H}_{2} \mathrm{O}$-acetone (mass ratio 3/7) for several times. Then, the $\mathrm{SiO}_{2}$ nanoparticles inside the polymer spheres were removed by adding the concentrated sodium hydroxide aqueous solution. The solution with a mass composition of 10.0 $\mathrm{NaOH}$ : 1.0 polyurea/ $\mathrm{SiO}_{2}$ composite microspheres was stirred at $30{ }^{\circ} \mathrm{C}$ for $4 \mathrm{~h}$ using the oil-bath system to corrode $\mathrm{SiO}_{2}$ nanoparticles. The final resulted microspheres were separated by centrifugation, washed with $\mathrm{H}_{2} \mathrm{O}$-acetone mixture, and dried at $70{ }^{\circ} \mathrm{C}$ in an oven. Then, $0.06 \mathrm{~g}$ of PPUM and $1.22 \times 10^{-5} \mathrm{~mol}$ of the $\mathrm{PyCOOH}$ was mixed into $30 \mathrm{~mL}$ of ethyl acetate, and stirred for $3 \mathrm{~h}$ at $40{ }^{\circ} \mathrm{C}$.

They were centrifugal separated, washed with ethyl acetate for several times. They are finally well dispersed in $10 \mathrm{~mL}$ ethyl acetate, preparing for investigating the fluorescent properties next step.

\section{Fluorescent properties of the material}

For the fluorescent study, the above PyCOOH-decorated PPUM dispersed in ethyl acetate was ultrasonically before testing. In a typical measurement for the fluorescent stability study, $1 \mathrm{~mL}$ of the suspension was removed from the above $10 \mathrm{~mL}$ PyCOOHdecorated PPUM, added into the quartz cuvette, and $0.1 \mathrm{~mL}$ of the solvent was injected every time. The ratio of the solvent (mol) and PPUM (g) increased to $4.7: 1$. The mixture were shaken and then kept static for $3 \mathrm{~min}$ before measuring. In a typical measurement for the controllable fluorescent properties study, $1 \mathrm{~mL}$ of the above suspension was diluted 12500 times by ethyl acetate, added into the quartz cuvette, and $100 \mu \mathrm{L}$ or $200 \mu \mathrm{L}$ of the aromatic compound $\left(9.562 \times 10^{-3} \mathrm{~mol} \mathrm{~L}^{-1}\right)$ was injected every time. The final ratio of the aromatic compounds (mol) and PPUM (g) was achieved to 90.6 : 1. The mixture were also shaken and then kept static for 3 min before measuring.

\section{The pH tolerance of the material}

The solution exhibiting various $\mathrm{pH}(1.6,4.0,6.8,8.7$ and 12.2) was obtained by adding different amounts of sodium hydroxide and hydrochloric acid in water respectively. Then, $0.06 \mathrm{~g}$ of the PPUM and $2 \mathrm{~mL}$ of the above solution was mixed together in a bottle, and ultrasonic-treated to make the PPUM welldispersed. Finally, the bottle was sealed off and then placed in a water bath oscillator operating at 140 osc per min and $40{ }^{\circ} \mathrm{C}$ for $6 \mathrm{~h}$.

\section{Results and discussion}

\section{Synthesis and fluorescent property of the PyCOOH-decorated PPUM}

The synthetic procedure of PyCOOH-decorated PPUM was shown in Scheme 1 and $\mathrm{S} 1 . \dagger$ The polyurea/SiO $\mathrm{S}_{2}$ microspheres

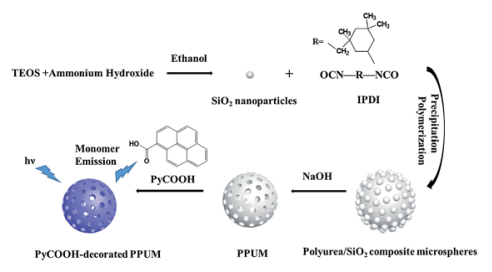

Scheme 1 Synthetic procedure of PyCOOH-decorated PPUM. 
were prepared by adding the uniform mono-dispersed $\mathrm{SiO}_{2}$ nanoparticles in isophorone diisocyanate (IPDI) via precipitation polymerization (Fig. 1a and b). Then, the $\mathrm{SiO}_{2}$ porogen was etched by $\mathrm{NaOH}$ solution and the PPUM host which exhibited 5 $\mu \mathrm{m}$ in diameter with the pore size of about $100 \mathrm{~nm}$ were obtained (Fig. 1c). To active the fluorescence of this porous host, the $\mathrm{PyCOOH}$ molecule was incorporated into the PPUM via electrostatic interaction and hydrogen bonding interaction between $\mathrm{PyCOOH}$ and pore wall (Fig. 1d). The loading capacity was also determined, in which $1 \mathrm{~g}$ of solid PPUM can load $0.216 \mathrm{mmol}$ of $\mathrm{PyCOOH}$. As shown in Fig. S2, $\uparrow$ the XRD patterns of PyCOOH-decorated PPUM exhibited a serious of diffraction peaks assigned to the PyCOOH and PPUM, suggesting the successful immobilization of the guest molecule on the porous host. The C1s, O1s and N1s peaks were found in the XPS survey spectra of PPUM and PyCOOH-decorated PPUM, suggesting that both of the samples are mainly composed of carbon, oxygen, and nitrogen elements (Fig. S3a †). ${ }^{29}$ The peak at binding energy of $531.2 \mathrm{eV}$ in the O1s spectrum of unmodified PPUM was assigned to the oxygen making a double bond with carbon (Fig. S3b $\dagger$ ), demonstrating the composition of polyurea. ${ }^{30}$ For the PyCOOH modified PPUM (Fig. S3b $†$ ), besides the peak at binding energy of $531.2 \mathrm{eV}$, the other peak $(532.6 \mathrm{eV})$ was attributed to the oxygen making single bonds with hydrogen or carbon, implying the immobilization of the guest PyCOOH on PPUM. ${ }^{30}$ The stability and $\mathrm{pH}$ tolerance of the composite material were also studied. Under the acid condition (pH: 1.6, 4.0 and 6.8), the surface of the composite sphere was not very smooth any more, indicating the corrosion happened (Fig. $\mathrm{S} 4 \dagger$ ). In the range of acidity, the extent of the damage became more serious with the decreasing $\mathrm{pH}$ value. However, this material was relative stable under the alkaline environment when the $\mathrm{pH}$ increased to 8.7 and 12.2 (Fig. S4†).

Interestingly, as shown in Fig. 2, the emission spectrum of the PyCOOH-decorated PPUM in solid state displayed strong peaks appearing at $380 \mathrm{~nm}$ and $398 \mathrm{~nm}$ assigned to the monomer emission of pyrene-based moiety, as well as a very
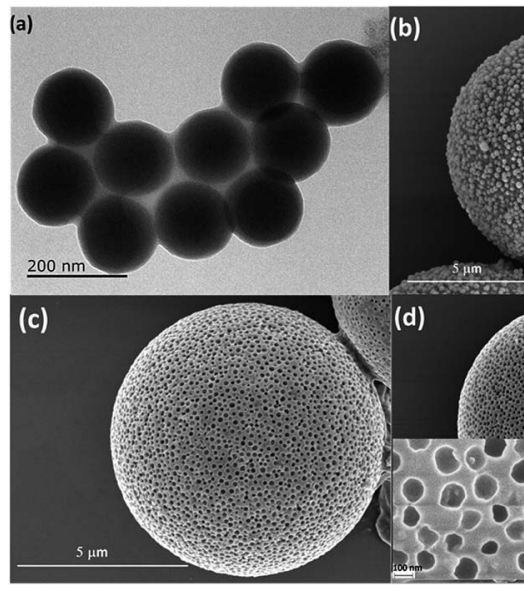

(d)
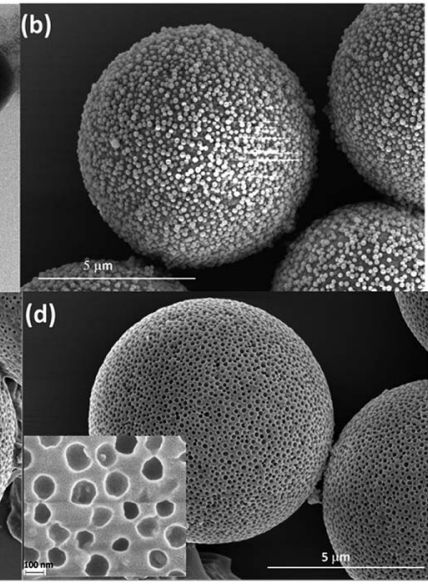

Fig. 1 TEM image of $\mathrm{SiO}_{2}$ nanoparticles (a); SEM images of polyurea/ $\mathrm{SiO}_{2}$ composite microspheres (b), PPUM (c) and PyCOOH-decorated PPUM (d and inset).

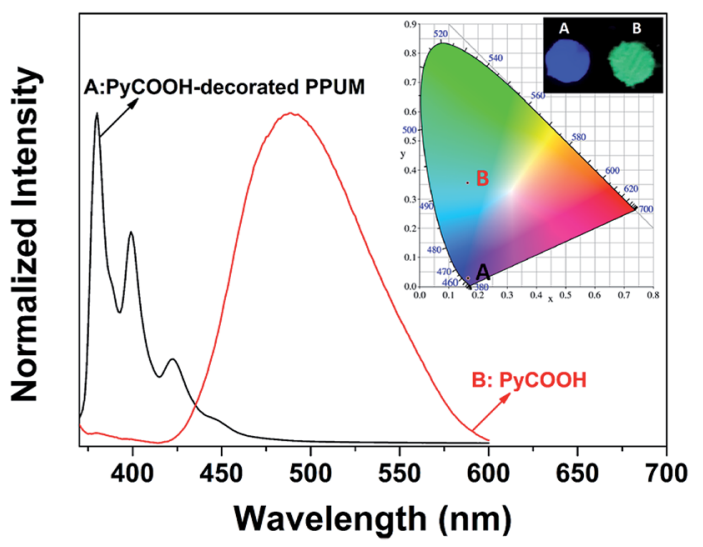

Fig. 2 Fluorescent spectra of solid $\mathrm{PyCOOH}$ and $\mathrm{PyCOOH}$-decorated PPUM excited at $345 \mathrm{~nm}$ (inset: 1931 CIE chromaticity diagram and digital photos under $365 \mathrm{~nm}$ UV light).

weak shoulder peak located at $422 \mathrm{~nm}$ which was indicative of the partially overlapped packing and conformational restriction of pyrenyl moiety, ${ }^{31}$ suggesting that the monomer emission of $\mathrm{PyCOOH}$ was mainly dominated. However, the pure $\mathrm{PyCOOH}$ with the same amount as the loading capacity of this host showed only broad band with the strong peak (about $480 \mathrm{~nm}$ ) which was resulted from the $\mathrm{PyCOOH}$ excimer, indicating that the PyCOOH generated a stable packing conformation with the enhancement of $\pi-\pi$ interaction. That is, the absence of the excimer emission in this solid PyCOOH-decorated PPUM demonstrated that the $\mathrm{PyCOOH}$ molecules were almost completely separated from each other by the porous host. It indicates that it is indeed an effective strategy to avoid the formation of excimers by using this 3-D PPUM host.

\section{Fluorescent stability of the PyCOOH-decorated PPUM dispersed in various organic solvents}

To further investigate the optical stability of this composite material in various solvents, changes in the fluorescence spectra by adding common organic solvents were examined (Fig. 3, S5 and S6†). The solvents exhibited different polarities and hydrophilic/hydrophobic properties, which could influence the dispersity and further induce the distinct fluorescence response. By adding most of the common organic solvents, the fluorescent intensity fluctuated slightly in the beginning, and finally reduced under high concentration. Interestingly, this material suffered from serious fluorescence quenching in the presence of DMSO due to the corrosion of the porous spheres, which was confirmed by the SEM image (inset of Fig. 3b). Most importantly, the fluorescence nearly remained unchanged upon the addition of ethyl acetate, indicated that this solvent was desirable to stabilize this microsphere (Fig. 3c). Comparing with the solid sample, the peaks of the monomer emission were red shifted to $385 \mathrm{~nm}(0,0)$ transition which was indicative of the unassociated fluorescence of $\mathrm{PyCOOH}, 404 \mathrm{~nm}(0,1)$ transition which was suggestive of the hydrogen bonding and $\pi-\pi$ interaction between adjacent PyCOOH molecules, and $425 \mathrm{~nm}(0,2)$ 

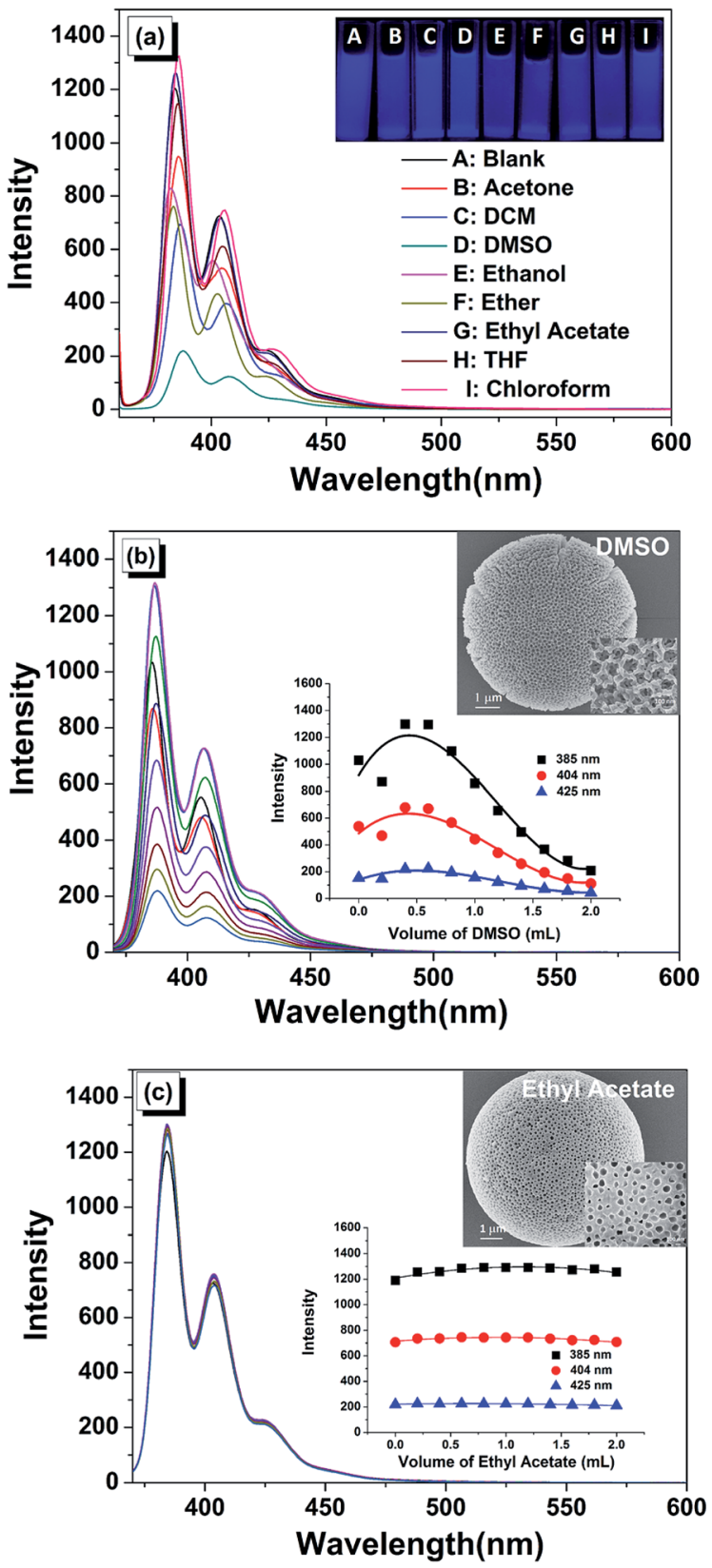

Fig. 3 Fluorescence spectra of PyCOOH-decorated PPUM upon the addition of various organic solvents $(2.0 \mathrm{~mL})$ excited at $345 \mathrm{~nm}$ (a). The fluorescent response by adding DMSO (b) and ethyl acetate (c). (Inset of (a): digital photos under $365 \mathrm{~nm}$ UV light; inset of (b) and (c): SEM images and fluorescent intensity of $385 \mathrm{~nm}, 404 \mathrm{~nm}$ and $425 \mathrm{~nm}$ under different volume of the solvents respectively).

transition which was assigned to restricted and overlapped pyrene moiety. ${ }^{32}$

\section{Controllable fluorescent properties of PyCOOH-decorated PPUM in the presence of different aromatic compounds}

To regulate the monomer emission of this prepared material, the fluorescent responses to a series of aromatic compounds were studied. The $m$-substituted aromatic compounds were chosen instead of its $p$ - and $o$-substituted isomers. Our aim is to utilize the different molecules to interact with this host-guest system and to induce the distinctly different fluorescent properties further, so the interference from its own structure to the fluorescent result should be minimized as far as possible. It was relatively difficult for the $p$-substituted compound to move into the pores due to the steric hindrance. Moreover, for the $o$ substituted compound, the possible intermolecular hydrogen bonds built between the $o$-substituted groups could affect the activity to interact with the host skeleton or guest molecule. Therefore, the study was mainly focused on inducing the different emission by the addition of the toluene based aromatic compounds containing different $m$-substituted groups (Fig. 4a, S7 and S8†).

Strikingly, in the presence of the $m$-cresol, the emission spectrum exhibited enhanced fluorescent intensity, as well as a distinctly different emission appearance comparing with the original blank sample, implying the microenvironment of $\mathrm{PyCOOH}$ was sensitive to $m$-cresol (Fig. 4b). However, the toluene without $-\mathrm{OH}$ group and phenol without $-\mathrm{CH}_{3}$ group behaved as fluorescent quencher and induce the decreased fluorescence without any change of peak shape (Fig. S7a and S7b $\dagger$ ). It is worth noting that the white suspension of the blank sample changed to a clearer solution gradually upon the addition of $m$-cresol, which was never observed in the presence of all the related compounds. We considered that this dramatic phenomenon implied that the sample was dissolved in $\mathrm{m}^{-}$ cresol. ${ }^{22,23}$ To further understand this phenomenon, the IR spectra of the PyCOOH-decorated PPUM in the absence and presence of $m$-cresol were performed (Fig. S9 $\dagger$ ). In the IR spectrum of PyCOOH-decorated PPUM, the strong adsorption peak at $3361 \mathrm{~cm}^{-1}$ and $1560 \mathrm{~cm}^{-1}$ were assigned to $\mathrm{NH}$ stretching vibration and $\mathrm{NH}$ plane-banding vibration respectively, and the peak at about $1640 \mathrm{~cm}^{-1}$ was attributed to urea carbonyl. ${ }^{25}$ For the sample in the presence of $m$-cresol, the above characteristic groups were also observed in the IR spectra besides the absorption peaks belonged to the residual $m$-cresol, indicating that the composition of the product after treating with $m$-cresol still mainly included the polyurea. However, these peaks shifted to higher frequency $\left(3372 \mathrm{~cm}^{-1}, 1589 \mathrm{~cm}^{-1}\right.$ and about $1700 \mathrm{~cm}^{-1}$ assigned to $\mathrm{NH}$ stretching vibration, NH planebanding vibration and urea carbonyl, respectively) comparing with the peaks in the IR spectrum of PyCOOH-decorated PPUM, demonstrating the weakened hydrogen bonding between polyurea ${ }^{33}$ In addition, the changes of the morphology during this process was confirmed by the SEM images, in which the porous skeleton can be damaged by adding very low amount of $m$-cresol $\left(4.30 \times 10^{-5} \mathrm{~mol} \mathrm{~L}^{-1}\right)$ and it disappeared finally $(5.80 \times$ $10^{-3} \mathrm{~mol} \mathrm{~L}^{-1}$ ) (Fig. S10†). According to the above results, we can deduce that the original strong hydrogen bonding interactions which distributed in every directions around the polyurea core could be weakened when the $m$-cresol molecules diffused between the polymer chains and the spherical shape with the regular pore was seriously damaged. We consider that the spheres probably transformed to a kind of linear polymers with the characteristic structure of polyurea when a full dissolution of the microspheres occurred. As a results, the guest PyCOOH molecules were no longer localized in the pore and they were 

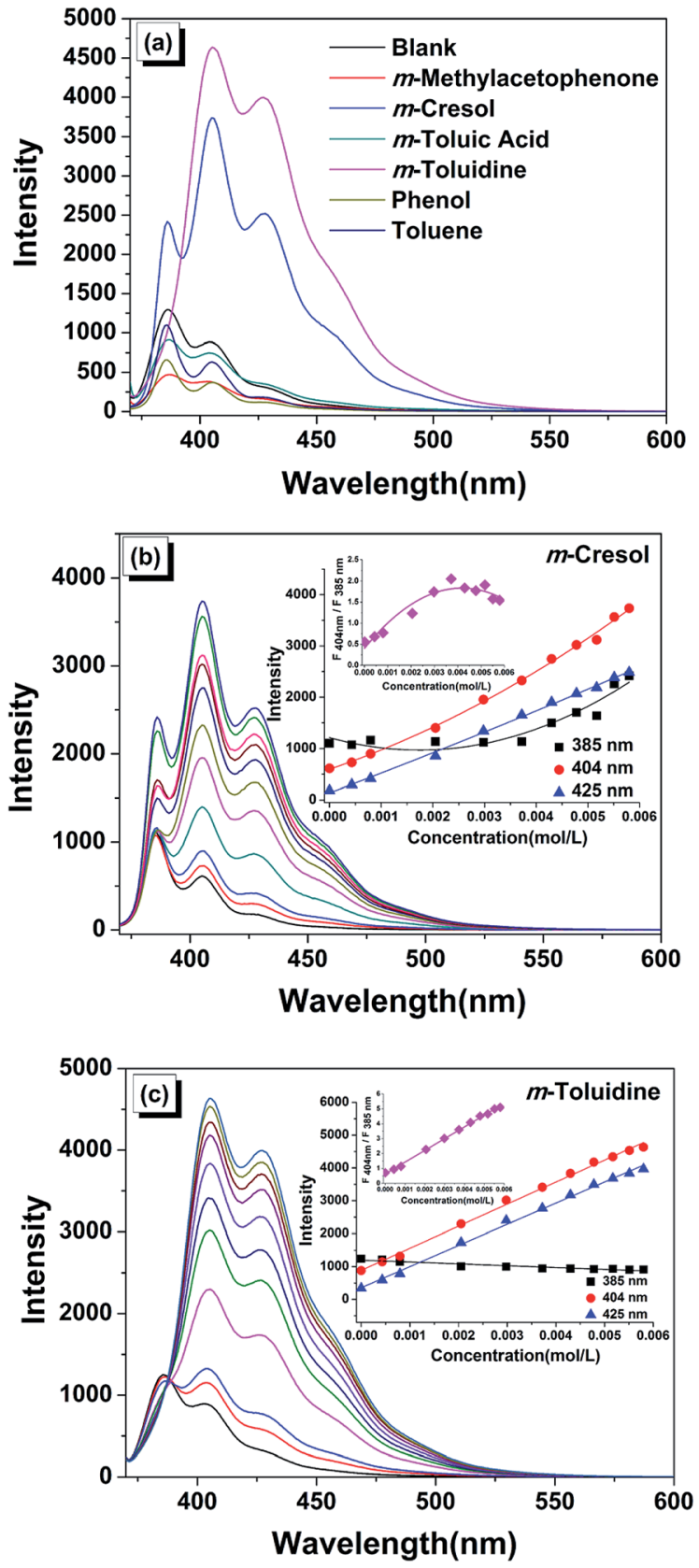

Fig. 4 Fluorescence spectra of $\mathrm{PyCOOH}$-decorated PPUM upon the addition of various aromatic compounds excited at $345 \mathrm{~nm}(5.80 \times$ $10^{-3} \mathrm{~mol} \mathrm{~L}^{-1}$ ) (a). The fluorescent response by adding the $m$-cresol (b) and $m$-toluidine (c). The concentration of the aromatic compounds increased from $4.30 \times 10^{-4} \mathrm{~mol} \mathrm{~L}^{-1}$ to $5.80 \times 10^{-3} \mathrm{~mol} \mathrm{~L}^{-1}$ (inset of (b) and (c): fluorescent intensity of $385 \mathrm{~nm}, 404 \mathrm{~nm}$ and $425 \mathrm{~nm}$ under different concentration of $m$-cresol and $m$-toluidine respectively; the intensity ratio of the peaks appearing at $404 \mathrm{~nm}$ and $385 \mathrm{~nm}$ ).

attached on the polyurea with linear structure (Fig. 5). That is, the microenvironment of the $\mathrm{PyCOOH}$ had changed during the addition of the $m$-cresol, in which it contacted with large amount of the hydrophobic carbon chains. As seen in emission spectrum, the enhanced monomer intensity suggested that pyrene moiety encountered a more hydrophobic environment, ${ }^{34}$ which was consistent with the dissolution process mentioned

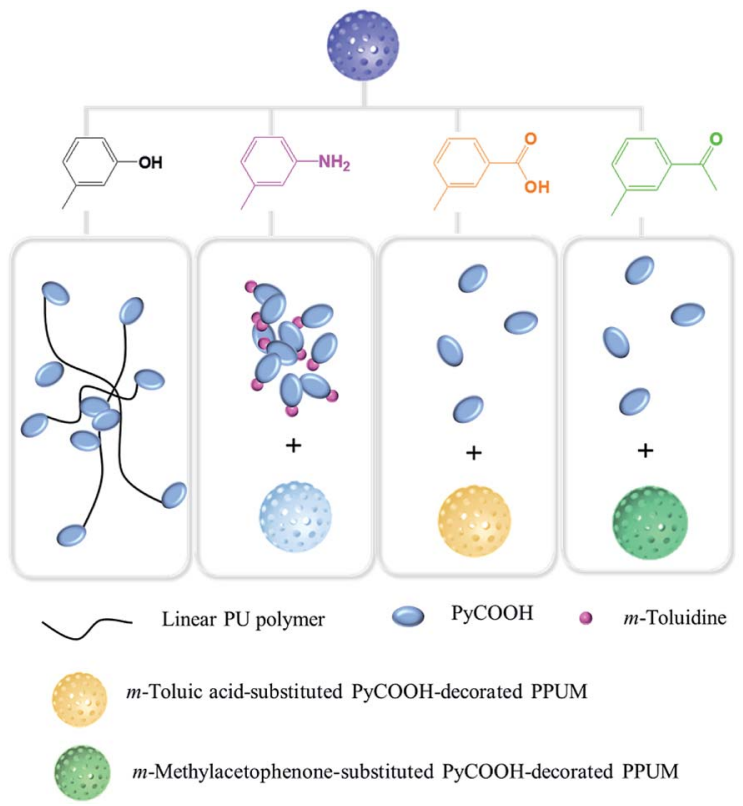

Fig. 5 Possible mechanism of the different fluorescent responses by adding various aromatic compounds.

above. Notably, the attachment of the $\mathrm{PyCOOH}$ on the flexible linear polymer in the dissolution process resulted in the enhanced interaction of the neighbouring $\mathrm{PyCOOH}$, which was confirmed by the fact that the value of $I_{404 \mathrm{~nm}} / I_{385} \mathrm{~nm}$ increased from 0.56 to a value higher than $1.0(1.32)$ when the concentration of the $m$-cresol achieved to $2.05 \times 10^{-3} \mathrm{~mol} \mathrm{~L}^{-1}$ (inset of Fig. 4b). However, the value achieved a level-off and then decreased slightly, because the PPUM skeleton almost disappeared when the amount of $m$-cresol increased to a certain high level. More excitingly, there was a growing tendency for the emission intensity at $475 \mathrm{~nm}$, suggesting that the possibility of generating the excimers increased.

Interestingly, the increased fluorescent intensity of $\mathrm{PyCOOH}-$ decorated PPUM by adding $m$-cresol was different from the previous results related to the decreased fluorescence in the presence of DMSO, although both of the samples were damaged. As mentioned in the experimental section, different from the solvent-composite PPUM system, the diluted $\mathrm{PyCOOH}-$ decorated PPUM was mixed with large amount of $m$-cresol in aromatic compound-composite PPUM system. When the amount of the DMSO and composite PPUM keep the same value as that of $m$-cresol and composite PPUM, the fluorescent intensity also decreased, however, the decreasing degree was lower compared with the fluorescence detected in the original solvent- composite PPUM system (Fig. S11a†). Similar as $m$ cresol, DMSO also induced the guest PyCOOH departed from the damaged skeleton and encountered more hydrophobic environment. However, the increase of the fluorescence induced by the corrosion of this skeleton was too low to compensate for the fluorescence quenching, so the fluorescent intensity didn't increase and the observed fluorescence quenching was not very obvious. Compared with the disappearance of the spheres in $m$-cresol system, the irregular 
polymer core and damaged spheres can be still observed even when the DMSO was excessive $(90.6: 1)$ shown in Fig. S11b. $\dagger$ That is, these distinct different properties of the composited materials were clearly observed by adding the DMSO and $m$ cresol, including dissolution and fluorescence, implying that the composite material is sensitive to $m$-cresol and it may have potential application in the discrimination of $m$-cresol.

Besides, when the $-\mathrm{OH}$ group is changed to $-\mathrm{NH}_{2}$ group $(\mathrm{m}$ toluidine), a different enhanced monomer emission with the dominant peak at $404 \mathrm{~nm}$ was obtained (Fig. 4c). When the positive $m$-toluidine containing amine group was added, large amounts of PyCOOH molecules might depart from the pores of PPUM and attach on the $m$-toluidine due to the much stronger electrostatic interactions between amino groups of $m$-toluidine and carboxyl group of $\mathrm{PyCOOH}$. Almost $\mathrm{PyCOOH}$ released from the pores and encountered a more hydrophobic outer surface area of the sphere, leading to the increasing intensity of monomer emission. As shown in Fig. 5, comparing with the arrangement of the PyCOOH which was supported by the long linear polymer in the presence of $m$-cresol, the distance between PyCOOH in the presence of $m$-toluidine might be shorter. As a result, the intensity of $385 \mathrm{~nm}$ assigned to the unassociated fluorescence of PyCOOH nearly disappeared, the intensity of $404 \mathrm{~nm}$ and $425 \mathrm{~nm}$ attributed to the interaction between PyCOOH became obvious, as well as the intensity of peak at about $475 \mathrm{~nm}$ related to the growing tendency for forming the excimers of PyCOOH increased.

Moreover, similar as the fluorescence in the presence of phenol and toluene, the reduced intensity by the addition of the $m$-toluic acid and $m$-methylacetophenone was observed (Fig. S7c, S7d, $\dagger$ and 5). However, $I_{404} \mathrm{~nm} / I_{385} \mathrm{~nm}$ increased comparing with the blank sample, implying that the $\mathrm{PyCOOH}$ rearranged towards the stronger interaction between them. The $m$-toluic acid with carboxyl group could lead to the substitution process, in which the $\mathrm{PyCOOH}$ was partially replaced by this aromatic acid due to the electrostatic interaction between the $m$ toluic acid and pore wall. However, as a weak aromatic acid, the $m$-toluic acid didn't have much advantage in competing with PyCOOH to attach on the pore wall of host and only small amount of $\mathrm{PyCOOH}$ located on the outer pore were substituted by the $m$-toluic acid. That is, the amount of $\mathrm{PyCOOH}$ released from the host was not enough to increase the fluorescent intensity, although some of them encountered a more hydrophobic surrounding. Similarly, the decreasing monomer emission and increasing $I_{404} \mathrm{~nm} / I_{385} \mathrm{~nm}$ was also induced by $m$ methylacetophenone. However, comparing with the substitution process in the presence of $m$-toluic acid, smaller amount of PyCOOH could be substituted by $m$-methylacetophenone, because the carbonyl group could interact with the pore wall via weaker hydrogen bonding, which was agree with the seriously reduced fluorescent intensity.

\section{Conclusions}

In summary, a novel pyrene derivative based composite material has been synthesized, in which the $\mathrm{PyCOOH}$ are immobilized in the pores of porous polyurea microspheres. Notably, its fluorescent spectrum only show strong monomer emission of $\mathrm{PyCOOH}$ due to that the guest molecule is isolated by the porous skeleton. Moreover, this material exhibited good fluorescent stability in some organic solvents, especially in ethyl acetate. Excitingly, the fluorescent properties can be regulated by adding various $m$-substituted aromatic compounds. When $m$-cresol was added, the strengthened intensity of monomer emission was caused by the unusual dissolution of this material. By adding the $m$-toluidine, the $\mathrm{PyCOOH}$ depart from the pores and connect with $m$-toluidine, resulting in the increasing monomer emission without the peak assigned to the unassociated $\mathrm{PyCOOH}(385 \mathrm{~nm})$. The attenuated monomer emission is resulted from the substitution of $\mathrm{PyCOOH}$ by $m$-toluic acid and $m$-methylacetophenone respectively, but more obvious decreased intensity was observed in the presence of latter molecule. All the results indicate that the $m$-substituted group of the aromatic compound has great influence on the fluorescent properties in this system. This reported discovery has provided a new insight into the tuneable fluorescence of pyrene derivative decorated composite material.

\section{Conflicts of interest}

There are no conflicts to declare.

\section{Acknowledgements}

This work is supported by the National Natural Science Foundation of China (No. 21601107).

\section{Notes and references}

1 Z. Xu, N. J. Singh, J. Lim, J. Pan, H. N. Kim, S. Park, K. S. Kim and J. Yoon, J. Am. Chem. Soc., 2009, 131, 15528.

2 K. C. Stylianou, R. Heck, S. Y. Chong, J. Bacsa, J. T. A. Jones, Y. Z. Khimyak, D. Bradshaw and M. J. Rosseinsky, J. Am. Chem. Soc., 2010, 132, 4119.

3 T. M. Figueira-Duarte and K. Mullen, Chem. Rev., 2011, 111, 7260.

4 J. Huang, Y. Wu, Y. Chen, Z. Zhu, X. Yang, C. J. Yang, K. Wang and W. Tan, Angew. Chem., Int. Ed., 2011, 50, 401.

5 T. Nokami, T. Matsuo, Y. Inatomi, N. Hojo, T. Tsukagoshi, H. Yoshizawa, A. Shimizu, H. Kuramoto, K. Komae, H. Tsuyama and J.-I. Yoshida, J. Am. Chem. Soc., 2012, 134, 19694.

6 K. Zhong, B. Guo, X. Zhou, K. Cai, L. Tang and L. Jin, Prog. Chem., 2015, 27, 1230.

7 H. Ma, F. Li, P. Li, H. Wang, M. Zhang, G. Zhang, M. Baumgarten and K. Muellen, Adv. Funct. Mater., 2016, 26, 2025.

8 X. Zhang, S. Wang and G. Xing, ACS Appl. Mater. Interfaces, 2016, 8, 12007.

9 S. Chanmungkalakul, V. Ervithayasuporn, S. Hanprasit, M. Masik, N. Prigyai and S. Kiatkamjornwong, Chem. Commun., 2017, 53, 12108.

10 W. Lu, J. W. Zhang, Y. J. Huang, P. Theato, Q. Huang and T. Chen, ACS Appl. Mater. Interfaces, 2017, 9, 23884. 
11 Y. Upadhyay, T. Anand, L. T. Babu, P. Paira, G. Crisponi, A. S. K. Kumar, R. Kumar and S. K. Sahoo, Dalton Trans., 2018, 47, 742.

12 Y. Gao, T. Ma, Z. Ou, W. Cai, G. Yang, Y. Li, M. Xu and Q. Li, Talanta, 2018, 178, 663.

13 T. Xiao, F. Wang, Y. Chen, X. Yang, T. Wei, C. Liu, S. Chen, Z. Xu, J. Yoon and X. Chen, Dyes Pigm., 2019, 163, 227.

14 S. Long, L. Miao, R. Li, F. Deng, Q. Qaao, X. Liu, A. Yan and Z. Xu, ACS Sens., 2019, 4, 281.

15 N. Hafezi, J. M. Holcroft, K. J. Hartlieb, E. J. Dale, N. A. Vermeulen, C. L. Stern, A. A. Sarjeant and J. F. Stoddart, Angew. Chem., Int. Ed., 2015, 54, 456.

16 H. Shionari, Y. Inagaki, K. Yamaguchi and W. Setaka, Org. Biomol. Chem., 2015, 13, 10511.

17 Y. Matsuo, K. Hatase and Y. Sugie, Chem. Commun., 1999, 43. 18 W. He, X. Gu and S. Liu, Adv. Funct. Mater., 2012, 22, 4023. 19 X. Jiang, X. Zhu and X. Z. Kong, Chem. Eng. J., 2012, 213, 214. 20 F. Zhang, X. Jiang, X. Zhu, Z. Chen and X. Z. Kong, Chem. Eng. J., 2016, 303, 48.

21 J. Xu, H. Han, L. Zhang, X. Zhu, X. Jiang and X. Z. Kong, RSC $A d v ., 2014,4,32134$.

22 X. Jiang, Y. Yu, X. Li and X. Z. Kong, Chem. Eng. J., 2017, 328, 1043.

23 X. Jiang, X. Li, X. Zhu and X. Z. Kong, Ind. Eng. Chem. Res., 2016, 55, 11528.
24 X. Z. Kong, W. Jiang, X. Jiang and X. Zhu, Polym. Chem., 2013, 4, 5776.

25 B. Yu, Y. Luo, H. Cong, C. Gu, W. Wang, C. Tian, J. Zhai and M. Usman, $R S C$ Adv., 2016, 6, 111806.

26 E. Miller and D. Jozwik-Styczynska, Spectrochim. Acta, Part A, 2009, 72, 312.

27 M. F. Pistonesi, M. S. Di Nezio, M. E. Centurion, A. G. Lista, W. D. Fragoso, M. J. C. Pontes, M. C. U. Araujo and B. S. Fernandez Band, Talanta, 2010, 83, 320.

28 W. Stober, A. Fink and E. Bohn, J. Colloid Interface Sci., 1968, 26, 62 .

29 Y. K. Song, Y. Y. Wu, H. T. Wang, S. Liu, L. Song, S. Li and M. Q. Tan, Food Chem., 2019, 293, 387.

30 F. Ahimou, C. J. P. Boonaert, Y. Adriaensen, P. Jacques, P. Thonart, M. Paquot and P. G. Rouxhet, J. Colloid Interface Sci., 2007, 309, 49.

31 M. Teng, X. Jia, X. Chen, Z. Ma and Y. Wei, Chem. Commun., 2011, 47, 6078.

32 B. H. Milosavljevic and J. K. Thomas, J. Phys. Chem., 1988, 92, 2997.

33 H. Han, S. Li, X. Zhu, X. Jiang and X. Z. Kong, RSC Adv., 2014, 4, 33520.

34 T. Zhang, S. D. Taylor, M. Palmer and J. Duhamel, Biophys. J., 2016, 111, 1267. 